\title{
RELATION OF LIGHT CURVE BEHAVIOUR WITH ACCRETION RATES IN BLACK HOLE CANDIDATE GRS1915+105
}

\author{
SANDIP K. CHAKRABARTI, A. NANDI AND S. G. MANICKAM \\ S.N. Bose National Centre for Basic Sciences, JD-Block, Salt Lake, Calcutta 700098 \\ E-mail: chakraba@boson.bose.res.in,anuj@boson.bose.res.in and \\ sivman@boson.bose.res.in
}

We report some properties of the light curves of GRS 1915+105 and present their

interpretation using the variation of net accretion rate.

Proceedings of 9th Marcel grossman Conference (Ed. R. Ruffini)

Recently, Belloni et all classified all light curves based on energy channels available in RXTE data. However, one needs to use channels dictated by physical considerations. When matter accretes in Keplerian and sub-Keplerian components, the centrifugal barrier heats up and puffs up the disk close to the hole. The hot electrons Comptonize intercepted soft photons $(\sim 2-3 \mathrm{keV})$ emitted by the Keplerian disk. Power-law hard photons pivot at $\sim 17-18 \mathrm{keV}$ and the number of photons with even higher energy is fewer. Thus, we separate the photons in $\mathrm{A}=0-3 \mathrm{keV}, \mathrm{B}=3-17 \mathrm{keV}$ and $\mathrm{C}=17-60 \mathrm{keV}$. Fig. 1 shows all types of 'softness' ratio diagrams plotted with $A / C$ along $\mathrm{Y}$-axis and $B / C$ along the $\mathrm{X}$-axislo. The numbered sequence is same as the sequencel $\chi, \alpha, \nu, \beta, \lambda, \kappa, \rho, \mu, \theta, \delta, \gamma$ and $\phi$ respectively. Here, Y-axis roughly measures the accretion rate of the Keplerian disk and $\mathrm{X}$-axis roughly measures a combination of the net accretion rate and geometry of the puffed up region (actually, soft photons intercepted by it). Our softness ratio is basically a collection of straight lines with slopes varing from class to class, clearly indicating that two component flows are essentiall and that the Keplerian and sub-Keplerian flows are not of constant ratio. Spectrum for cases in Panel 1 is Hard, that of Panel 9 is Semi-Soft, those of Panels 10-12 are Soft and the rest belongs to Intermediate classes where transitions from low to high count take place 3 .

Belloni et all pointed out that there are three basic states (A, B and C). Following Chakrabarti et al 5 - we rename them to States HW (Hard state with Winds), CC (Compton cooled sonic sphere) and EA (Enhanced Accretion) respectively. In each class of the light curve, several States may be observed. While these states correspond to three 'hazy' areas (banana, atoll, Z-shaped etc.) in hardness ratio diagram $\mathrm{Z}$, according to our choice, for a given class, they roughly correspond to straight lines (Fig. 1), only slopes vary from class to class. In Fig. 2a, we fit lines in classes $\kappa, \lambda, \beta$ and $\theta$. Dots represent $\mathrm{HW}\left(\mathrm{C}^{2}\right)$ and circles represent $\mathrm{CC}(\mathrm{A})$ and squares are mixtures of CC (A) and EA (B). Slopes of straight lines in HW states in $\kappa, \lambda$ and $\theta$ are $0.137,0.055$ and 0.045 respectively. In Fig. 2b, correlation of duration of QPOs with QPO frequencies are plotted 6 for $\theta, \kappa$ and $\lambda$ classes. When fitted 6 the accretion factor $\Theta_{\text {dotm }}$ follows the same sequence as the slopes given above. Thus these two figures independently give handles on accretion rates. In Fig. 2c, dots, small circles, triangles and squares represent HW (C), CC (A), EA (B) and mixtures of CC (A) and EA (B) respectively. Dotted boundaries confine 


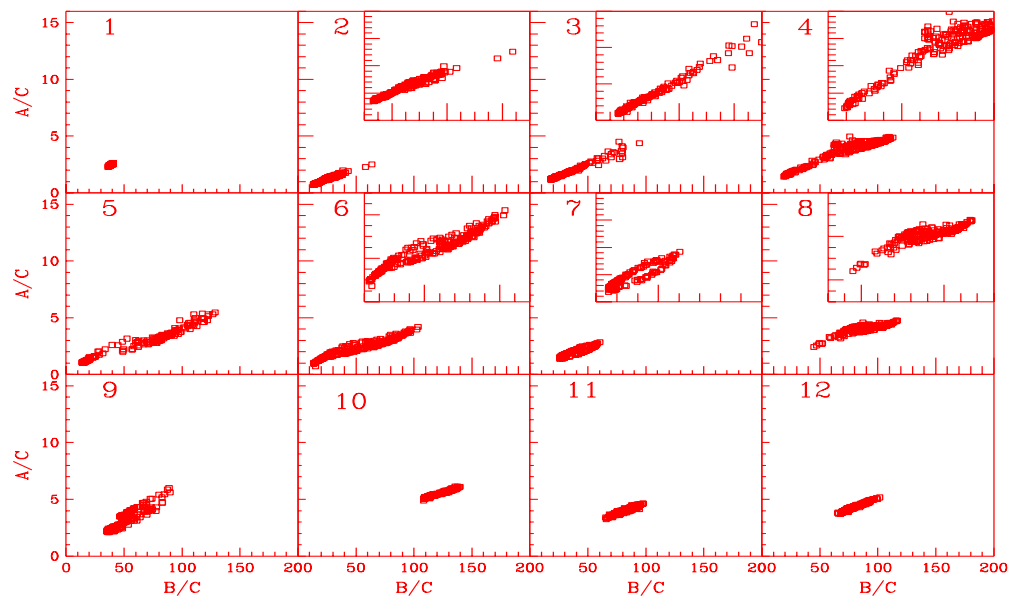

Figure 1. Softness Ratios A/C vs. B/C plotted for all the 12 types of light curves.
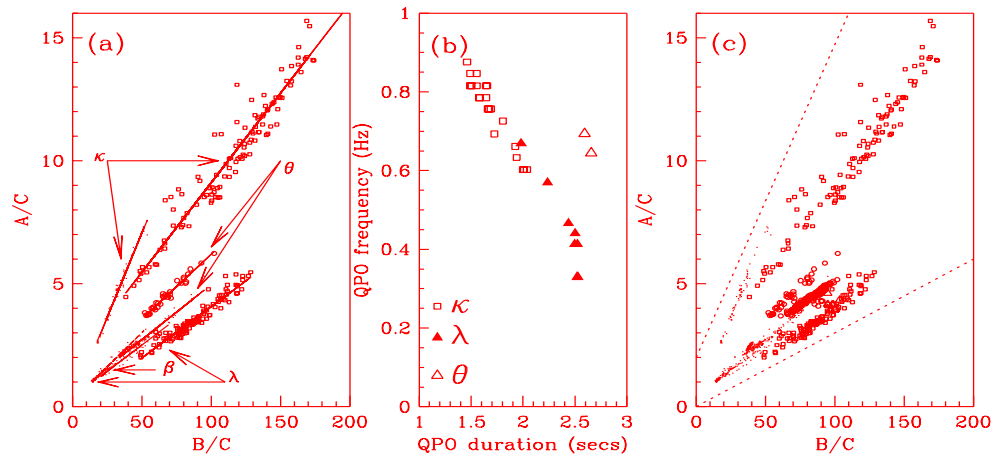

Figure 2. (a) Softness ptio diagrams using our 'paradigm' recommended channels for $\kappa, \beta, \lambda$ and $\theta$ classes of Belloni et all . (b) Chakrabarti-Manickam correlation for these classes show progressive rise of normalization from $\theta$ to $\lambda$ to $\kappa$ types. (c) State-wise separation of the parameter space.

softness ratios. Variation of slopes of various classes of light curves in a given state is the proof that a pure Keplerian disk, or a pure ion pressure supported Compton cloud cannot explain the observations and one definitely requires two component accretions flows 1 as demanded by advective disk paradigm.

\section{References}

1. S.K. Chakrabarti and L.G. Titarchuk, Astrophys. J., 455, 623 (1995).

2. T. Belloni et al. Astron. Astrophys. 355271 (2000).

3. A. Nandi, S.G. Manickam and S.K. Chakrabarti Ind. J. Phys. 74B, 331 (2000).

4. S.K. Chakrabarti, S.G. Manickam, A. Nandi and A.R. Rao (this volume).

5. S.K. Chakrabarti and A. Nandi, Ind. J. Phys. (2000) in press.

hale: submitted to World Scientific on November 6, 2018 
6. S. K. Chakrabarti and S. G. Manickam, Astrophys. J. 531, L41 (2000). 